François-Emmanuel BoUCHER, «1723-1815 : le genre épique en évolution », @nalyses, hiver 2006

\title{
François-Emmanuël BoucHER
}

\section{3-1815 : le genre épique en évolution}

Voilà un livre bien documenté (Roulin a répertorié plus de quatre-vingts poèmes épiques de 1723 à 1815), qui a comme point de départ l'idée que l'épopée est un genre multiforme dont les règles évoluent, de sorte qu'elles deviennent difficilement repérables. Selon les époques, explique Roulin tout au long de son livre, l'épopée peut être composée en vers ou en prose. Elle chante parfois les premiers balbutiements d'une histoire où transparait le rêve d'un monde meilleur ou, encore, elle raconte un fait passé, révolu, mythique et fondateur. Au XVIII ${ }^{\mathrm{e}}$ siècle, plus précisément, selon les partis pris, l'épopée est un genre qui montre une France dont la gloire, incarnée par l'allégorie de la Liberté, est à l'aube de son développement ou, au contraire, au seuil de son crépuscule. En fait, comprendre l'épopée à cette période implique d'abord que l'on se dégage d'une définition générale afin de prendre en considération les multiples caractéristiques qui la traversent à chaque fois qu'elle module les bouleversements historiques.

\section{Voltaire, héritages et postérité}

Afin de mieux comprendre l'évolution et la spécificité du genre épique au XVIII ${ }^{\mathrm{e}}$ siècle, Rolin établit d'abord une comparaison entre les épopées virgiliennes et homériques. Comme le démontrent dans son livre de nombreuses analyses, certains auteurs, entre le début des Lumières et l'aventure napoléonienne, se positionnent soit dans la lignée du poète latin, soit dans celle du poète grec. Un peu comme Virgile se distinguerait du modèle homérique par la description du bouclier de son héros, les Lumières se distingueraient de leurs prédécesseurs de l'âge classique en faisant de l'épopée un lieu où l'on chante les faits héroïques à venir plutôt que de mettre en valeur des actions passées à l'intérieur d'un univers cosmogonique, clos et révolu. Chez Virgile, par exemple, les évocations traditionnelles de la genèse du monde et la description des travaux saisonniers furent remplacées par une illustration des hauts faits d'arme passés et de ceux à venir. 
François-Emmanuel BoUCHER, «1723-1815 : le genre épique en évolution », @nalyses, hiver 2006

Plutôt que de parler de l'influence des dieux sur le changement des saisons, il raconte l'histoire romaine de Romulus jusqu'au règne d'Auguste. Plutôt que d'appuyer son univers mythologique sur un univers clos, comme le faisait son modèle homérique, Virgile raconte une histoire qui évolue, qui bouscule, prometteuse par le fait même de transformations positives. Ce que Voltaire admire et imite, dans le récit virgilien, explique Roulin, est «un modèle où articuler histoire et politique, lieu de mémoire et vision prospective » (p. 17). En effet, avec La Henriade (1728), qui connut une soixantaine de rééditions du vivant de son auteur, l'épopée devient utopique (p. 61), en ce qu'elle s'intéresse à l'histoire passée comme à une leçon à assimiler pour inventer une civilisation nouvelle dont les vices seraient corrigés, si ce n'est supprimés par l'abolition de leurs causes. Par exemple, explique Roulin, dans sa Henriade, Voltaire raconte l'épisode de la Saint-Barthélemy dans le but de faire de cet épisode passé un contre-exemple pour l'histoire à venir et, ultimement, afin que son épopée devienne "une leçon de réflexion politique sur l'état de la France au début du règne de Louis $\mathrm{XV} »($ p. 66). L'épique devient véhicule de tolérance.

Une des ruptures fondamentales, remarque toujours l'auteur, dans l'évolution de l'épopée à partir de Voltaire, une des caractéristiques qui le distingue de ses prédécesseurs et qui marque les auteurs épiques qui suivront, est le recours non pas aux dieux de l'Antiquité païenne ni à la mythologie chrétienne, à chaque fois que l'on illustre les forces qui président à l'évolution humaine, mais à l'allégorie (p. 65). La Henriade, par exemple, met en scène de nouvelles muses telle «Uranie» («muse de l'astronomie », p. 111), la «Vérité » («inspiratrice qui se pose audessus de toute religion », p. 65), le «Génie de la Russie », l'«Éternelle Sagesse », la «Liberté » ou le «Peuple » («qui n'est pas pur instrument, mais qui peut aussi être la cause de l'événement. [...] L'homme bénéficie du libre-arbitre », p. 65). Le merveilleux, précise Roulin, n’est pas exclu de l'épopée, "mais donne une représentation de l'équilibre entre la part de l'homme et celle des dieux (ou de la Providence) dans la marche de l'histoire » (p. 40). Désormais, les transformations historiques dépendent de la Sagesse et de la Liberté, mères non pas de surhommes, mais de tous les êtres humains, filles non pas de l'athéisme mais de la religion naturelle. Bien qu'on eût apprécié davantage d'explication sur 
François-Emmanuel BoUCHER, «1723-1815 : le genre épique en évolution », @nalyses, hiver 2006

les contextes philosophique et théologique dans lesquels apparaissaient ces nouvelles divinités introduites dans l'épopée voltairienne, Roulin a bien vu, dans cette métaphore du sacré, non pas une laïcisation du genre, mais la fabrication d'un nouveau type de merveilleux.

Aussi cette invention de muses inédites devient-elle nécessaire en raison de la recherche de nouveaux thèmes, car le propre des sujets des épopées, depuis la Henriade, est d'être récent. Chez Voltaire, explique Roulin, «c'est un moyen pour la poésie de s'emparer de l'Histoire et d'offrir une vision politique » (p. 65). Si, comme l'indique l'auteur, les prophéties sont préférées, par les Lumières, aux récits des origines, La Henriade a ceci de particulier qu'elle dépasse la simple illustration du futur (comme le fait Virgile, par exemple, avec la description des images qui figurent sur le bouclier de son héros) : chez Voltaire, même la fiction du futur sert de point de vue critique sur l'histoire de la nation française et le devenir de son peuple (p. 79). Aux yeux de ce philosophe, c'est à l'homme et non aux dieux de faire l'histoire, c'est à lui à exercer son jugement sur les faits passés et surtout sur ceux à venir: son dieu «laisse aux hommes la liberté de leur destinée » et son épopée « s'empare de l'Histoire » pour offrir une portée politique à sa fiction poétique (p. 87).

Bien sûr, alors même qu'il innove par son approche poétique, Voltaire se positionne à l'encontre de plusieurs courants littéraires qui lui sont contemporains. Le plus traditionnel est bien sûr incarné par Le Bossu, qui, dans ses traités rhétoriques, "a réduit l'histoire à un exemplum moral » d'inspiration religieuse (p. 88). Un autre type d'épopée émerge aussi à l'époque de La Henriade où l'écrivain ne serait plus, à la manière de Voltaire, critique, savant ou philosophe, mais où il deviendrait prophète. «Extatiques» et «visionnaires» (p. 96), ces héros préfigurent déjà ce qui deviendra le héros romantique. Un Batteux (Cours de belles lettres distribué par exercices, 1747-1748), par exemple, explique Roulin, ou un Louis Racine (qui écrit, dans le cadre de sa traduction de Milton, un Discours sur le poème épique, à l'occasion des Remarques d'Addison sur celui de Milton, 1755) annoncent une nouvelle révolution du genre. Pour eux, la quête devient religieuse; au lieu du 
François-Emmanuel BoUCHER, «1723-1815 : le genre épique en évolution », @nalyses, hiver 2006

règne de la raison ou de la toute-puissance de la critique, apparait le désir du voyage, d'un autre monde, de l'initiation et de la mystique.

\section{Les épopées de la deuxième moitié du $\mathrm{XVIII}^{\mathrm{e}}$ siècle : influence de Voltaire et du Nouveau Monde}

Dans son article «Épopée» de L’Encyclopédie (1755), Marmontel affirme que l'action politique peut être contemporaine à l'époque du créateur, que cette action peut être dirigée par le peuple et, mieux encore, qu'elle peut être révolutionnaire et ainsi « devenir matière à épopée » (p. 105). Dans son Bélisaire (1767), par exemple, que certains qualifiaient, à l'époque, de «roman épique écrit en prose» (p. 105), Marmontel construit son récit de manière à véhiculer une morale civique, qui s'intéresse moins au rang des personnages qu'aux actions des individus (p. 105). La quête de valeurs qui s'y manifeste s'apparente à un rite religieux grâce auquel le héros se libère d'une société vouée à la dépravation. Mais outre ce type de récit qui, sur certains points, préfigure l'épisode révolutionnaire, la fin du XVIII siècle subit aussi une nouvelle sphère d'influence: celle de la découverte de l'Amérique, "sujet neuf dans l'épopée française » (p. 107). Souvent représenté comme une idylle qui n’a pas encore connu la corruption, la monarchie, les guerres de religion, le territoire fantasmagorique du Nouveau Monde devient dès lors un sujet de prédilection pour les épopées telles le Christophe Colomb on l'Amérique découverte (1773) de Nicolas-Louis Bourgeois, Le Nouveau Monde (1781) de Robert-Martin Le Suire et L'Amérique découverte (1782), épopée en prose de Pierre Laureau. Cette «mode» (p. 108), qui aboutira aux Natchez de Chateaubriand, œuvre achevée vers 1800, dont il publiera une " une version hybride, mi-roman, mi-épopée » en 1826, fera de l'épopée le contre-exemple de l'idéal voltairien.

En fait, ce qui intéresse davantage les auteurs épiques de cette nouvelle mode est la richesse de l'Amérique, «pays de l'abondance» (p. 109) où s'affrontent le « colon civilisé » et «l'indien sauvage » (p. 109). Les héros d'Amérique et les protagonistes de l'histoire du monde civilisé se disputent le fanatisme religieux, les auto-da-fés et les sacrifices humains (p. 115). Fait intéressant, insiste Roulin, les épopées écrites au tournant du XVIII ${ }^{\mathrm{e}}$ siècle ne s'entendent pas sur le sort du Nouveau et de l'Ancien monde : pour les uns, comme Le 
François-Emmanuel BoUCHER, «1723-1815 : le genre épique en évolution », @nalyses, hiver 2006

Suire, dans son poème Le Nonveau Monde (1781), Dieu se manifeste plus clairement chez les peuples qui sont près de la nature (p. 116); par contre, chez les autres, comme Marmontel dans Les Incas (1778), l'existence ne peut être possible que dans une société civilisée (p. 123), à condition bien sûr qu'elle repose sur une religion favorable au développement du genre humain. Cette vision deviendra plus pessimiste encore dans Les Natchez (1800) de Chateaubriand où, désormais, ni les lois arbitraires de la vie en société ni les conditions de survie difficiles dans l'état de nature ne seront idéalisables : aux lendemains de la Révolution, la «vision pessimiste du politique » (p. 198) est indissociable d'une impasse poétique.

\section{Nouvelle rupture : l'enthousiasme épique}

Dans le Supplément à l'Encyclopédie (1776), explique Roulin, l'article «Épopée » marque « un tournant radical par rapport à L'Encyclopédie » (p. 133). L'on définit désormais l'épopée moins par des règles strictes que par «l'enthousiasme» (p. 134). À l'instar du roman romantique, les épopées de la fin du XVIII ${ }^{\mathrm{e}}$ siècle ont aussi été marquées par la littérature anglaise, par la publication faite par Macpherson de fragments retrouvés des chants d'Ossian (1765), ainsi que par la publication des extraits des Eddas (1763) faite par Paul-Henri Mallet (p. 142). Chez les auteurs et les théoriciens du genre épique, écrit Roulin, qu'il s'agisse de Mme de Staël (De la littérature, 1800), de Parny (Isnel et Asléga, 1803) ou de Chateaubriand (Le Génie du christianisme, essai, 1802 et Les Martyrs, roman épique, 1809), ni l'Antiquité, ni l'Amérique ne servent plus de modèle à la civilisation européenne : le Moyen Âge devient maintenant la «source de la liberté moderne » (p. 142). L'on passe ainsi d'un genre qui chante les progrès du genre humain à une apologie de l'âge d'or : «l'épopée est peu à peu aspirée dans cette dynamique rétrospective, alors que Voltaire en avait fait le poème d'un monde meilleur à venir» (p.141). C'est cette nouvelle conception de l'épopée, "primitive, orale », en un mot, "romantique », conclut l'auteur, qui freinera la prospérité de La Henriade (p. 150). Un autre monde se met en place, dont la finalité n'est plus la tolérance ni la promotion des valeurs « améliorées » du monde antique. 
François-Emmanuel BoUCHER, «1723-1815 : le genre épique en évolution », @nalyses, hiver 2006

\section{Trêve napoléonienne?}

Même les épopées qui chantent ou déplorent le règne napoléonien s'inscrivent dans la lignée de l'influence des Poésies d'Ossian, en deviennent proprement les «avatars», selon Roulin (p. 166). Pour certains, comme Ménégault de Gentilly dans La Napoéide (1806), l'on peut décrire, grâce à la volonté divine, Napoléon comme l'héritier de l'empire de Charlemagne (p. 171); d'autres, comme Mme de Genlis dans Les Chevaliers du cygne, ou la Cour de Charlemagne (1795) ou Mme Cottin dans Mathilde, ou Mémoires tirés de l'bistoire des croisades (1805), préfèrent chanter la symbiose entre les idéaux hérités des révolutionnaires et leurs lointaines origines chez les chevaliers du Moyen Âge (p. 175). L'époque médiévale, explique Roulin, étant une idylle révolue, ayant une "essence atemporelle» (p. 177), l'épopée dans sa forme devient un lieu dans un espace clos, appartenant à un passé accompli sans horizon utopique. À la différence des Anciens, par contre, l'histoire médiévale n'est pas l'aperçu lumineux d'une guerre victorieuse et éclatante, mais le pâle segment d'une petite histoire insignifiante et désagrégée : «La grande épopée, celle de l'origine héroïque, est médiévale; l'Histoire moderne, désormais tragique ou sans finalité, est du côté du grotesque. Dès lors, on prend conscience que le devenir historique s'émiette, ressortit du fragment et s'appréhende, comme le montre La Légende des siècles, par de petites épopées »(p. 180). Cette modification poétique est indissociable de l'apparition d'un doute, du pouvoir des désenchantements.

\section{Chateaubriand ou l'aboutissement poétique}

Dans son chapitre consacré à l'œuvre de Chateaubriand, Roulin montre qu'un renouveau esthétique caractérise désormais l'épopée : la nature, perçue à travers l'influence d'Ossian, chantée à la manière rousseauiste, «devient un pivot essentiel de l'articulation entre poésie et Histoire » (p. 186). Il reste que dans Les Natchez, explique l'auteur, Chateaubriand ne parvient à idéaliser ni l'homme vivant à l'état de nature ni l'homme civilisé. Paradoxalement, il doit se détacher du modèle terrien, du retour aux origines, aux racines, à la nature, pour passer outre à son pessimisme radical et ainsi insuffler l'espoir à sa trame poétique: aux considérations politiques modernes, 
François-Emmanuel BoUCHER, «1723-1815 : le genre épique en évolution », @nalyses, hiver 2006

Chateaubriand préfère les bouleversements antiques qui ont assuré «le passage et le progrès de Rome à la France »; aux idées philosophiques qui caractérisent les Lumières et qui ont assuré la Révolution française, il substitue le christianisme qui est « né dans Rome » et qui assurera la libération du peuple français du joug du despotisme et des injustices (p. 204). Ainsi, comme l'explique Roulin, afin de se dégager d'un pessimisme auquel le confine l'impasse politique du présent, Chateaubriand choisit d'avoir recours aux sujets passés où le désastre implique les réalisations prophétiques d'un autre monde. Son but est de montrer comment ces sujets sont les garants non seulement « de la continuité historique » mais aussi « de la liberté moderne » (p. 205).

Ainsi, ayant figure de pivot dans l'histoire de l'épopée française, $L a$ Henriade de Voltaire est à la fois, aux yeux de Jean-Marie Roulin, l'aboutissement d'une tradition historique et littéraire qui remonte à l'Antiquité, et l'origine du bouleversement de cette même tradition qui subira dès lors maintes métamorphoses intimement liées au débat sur la nation qui marque le XVIII ${ }^{\mathrm{e}}$ siècle. Avec Les Natchez et Les Martyrs, Chateaubriand incarne les tendances les plus opposées du genre épique qui devient moins l'aboutissement des efforts d'un Voltaire que le crépuscule des possibilités du devenir d'un genre où les réalisations prophétiques se substituent à l'évolution de l'histoire. Roulin a raison de dire que le genre s'essouffle en France. En fait, il se déplace, comme le disait Peter Hentsch dans une perspective plus philosophique, vers l'est, vers le nouveau continent, vers l'Amérique ${ }^{1}$, et s'incarne dans de nouvelles formes esthétiques liées à un art beaucoup moins littéraire qu'audiovisuel, en somme franchement distinctes que celles qui ont inspiré le genre épique dans la littérature française.

Référence : Jean-Marie Roulin, L'Épopée de Voltaire à Chateaubriand: poésie, histoire et politique, Oxford, Voltaire Foundation, 2005, 278 p.

\footnotetext{
${ }^{1}$ Peter Hentsch, Raconter et mourir. Aux sources narratives de l'imaginaire occidental, Montréal, Presses de l'Université de Montréal, 2002.
} 